\title{
Genome-wide association study identifies candidate genes related to oleic acid content in soybean seeds
}

\author{
Xiaoyi Liu' ${ }^{1,2,3+}$, Di Qin ${ }^{2,3^{*}+}$, Angela Piersanti ${ }^{2}$, Qi Zhang ${ }^{3}$, Cristina Miceli ${ }^{2^{*}}$ and Piwu Wang ${ }^{3^{*}}$ (D)
}

\begin{abstract}
Background: Soybean oil is a complex mixture of five fatty acids (palmitic, stearic, oleic, linoleic, and linolenic). Soybean oil with a high oleic acid content is desirable because this monounsaturated fatty acid improves the oxidative stability of the oil. To investigate the genetic architecture of oleic acid in soybean seeds, 260 soybean germplasms from Northeast China were collected as natural populations. A genome-wide association study (GWAS) was conducted on a panel of 260 germplasm resources.

Results: Phenotypic identification results showed that the oleic acid content varied from 8.2 to $35.0 \%$. A total of 2, 311,337 single-nucleotide polymorphism (SNP) markers were obtained. GWAS analysis showed that there were many genes related to oleic acid content with a contribution rate of 7\%. The candidate genes Glyma.11G229600.1 on chromosome 11 and Glyma.04G102900.1 on chromosome 4 were detected in a 2-year-long GWAS. The candidate gene Glyma.11G229600.1 showed a positive correlation with the oleic acid content, and the correlation coefficient was 0.980, while Glyma.04G102900.1 showed a negative correlation, with a coefficient of -0.964 .
\end{abstract}

Conclusions: Glyma.04G102900.1 on chromosome 4 and Glyma.11G229600.1 on chromosome 11 were detected in both analyses (2018 and 2019). Glyma.04G102900.1 and Glyma.11G229600.1 are new key candidate genes related to oleic acid in soybean seeds. These results will be useful for high-oleic soybean breeding.

Keywords: Soybean, Oleic acid, SNPs, Genome-wide association study, Oleic acid-associated genes

\section{Background}

Soybean [Glycine $\max$ (L.) Merrill] originated in China and has been cultivated for more than 3000 years [1]. Soybean oil accounts for $20-25 \%$ of the total global fat and oil production and $30-35 \%$ of the total edible vegetable oil production [2]. In China, soybean oil is an important constituent of the diet, and it is considered a major factor in the maintenance of a healthy population. Soybean oil is a

\footnotetext{
* Correspondence: di.qin@unicam.it; 603988396@qq.com;

Cristina.Miceli@unicam.it; wangpiwu189@163.com

${ }^{+}$Xiaoyi Liu and Di Qin contributed equally to this work.

${ }^{2} \mathrm{~S} c h \mathrm{ool}$ of Biosciences and Veterinary Medicine, University of Camerino, Camerino, MC, Italy

${ }^{3}$ Biotechnology Center of Jilin Agricultural University, Jilin Agricultural University, Changchun 130118, PR China

Full list of author information is available at the end of the article
}

complex mixture of five fatty acids (palmitic, stearic, oleic, linoleic, and linolenic), all of which have different melting points, oxidative stabilities, and chemical functionalities [3].

The fatty acid composition of soybean oil is approximately 5 to $11 \%$ linolenic acid, 43 to $56 \%$ linoleic acid, 15 to $33 \%$ oleic acid, and 11 to $26 \%$ saturated acids [4]. Palmitic acid and stearic acid are saturated fatty acids that constitute $15 \%$ of soybean oil. In recent times, there has been a running debate, mainly in mainstream literature, regarding the effects of palmitic acid and stearic acid consumption on heart function, especially in the development of coronary artery disease [5, 6]. Linolenic and linoleic acids are polyunsaturated fatty acids that constitute $80 \%$ of soybean oil. Linolenic acid is needed for normal human growth and development and can lower the cholesterol 
content in blood, but this acid is not resistant to high temperatures. Atmospheric oxygen and ultraviolet rays can oxidize linolenic acid, resulting in the odour of soybean oil, which lowers the nutritional value of soybean oil. Oleic acid is a monounsaturated fatty acid, and soybean seeds with high oleic acid content also exhibited reduction in or elimination of chemical hydrogenation processes, reducing the cost of soybean oil processing [7]. The cultivation of soybean varieties with high oleic acid content has become an important goal of high-quality soybean breeding [8].

Genome-wide association analysis (GWAS) presents a powerful tool to connect this trait with the underlying genetics. With the rapid development of nextgeneration sequencing technology, GWAS has been successfully applied to plants such as rice and Arabidopsis [9, 10]. A large number of genetic variations associated with complex traits have been identified by the GWAS method [11]. In soybean, GWAS was performed to identify quantitative trait loci (QTLs) controlling seed oil concentration in 298 soybean germplasm accessions that exhibited a wide range of seed protein and oil content [12]. A soybean breeding germplasm population (279 lines) was established to perform a GWAS, and 8 QTLs were found that explained phenotypic variances ranging from 6.3 to $26.3 \%$ [13]. These results demonstrated that the use of GWAS with specially designed mapping populations is effective in uncovering the basis of key agronomic traits.

Scientists have successfully used the GWAS method to obtain a large number of candidate genes [14-16]. However, after the discovery of new candidate genes, verifying their biological function becomes a hot topic of research; RNA interference technology, the establishment and application of biochips, real-time fluorescent quantitative PCR technology (qRT-PCR), and gene editing technology provide, theoretically, a basis for candidate gene function verification. In this study, we selected two key candidate genes and measured their expression in four different tissues (root, stem, leaf and seed) of 14 diverse soybean lines appropriately selected using qRT-PCR.

Specifically, 260 soybean germplasms from Northeast China (Heilongjiang Province, Jilin Province and Liaoning Province) were collected as natural populations. The soybean lines were planted in the field of Jilin Agricultural University from 2018 to 2019. The fatty acid content in the soybean seeds as determined by a NIRS DS 2500 instrument after harvest. Specific-locus amplified fragment sequencing (SLAF-seq) technology was used to sequence the genomes of the 260 soybean materials, and GWAS was used to screen candidate genes related to soybean oleic acid content.

\section{Results}

Phenotypic identification of oleic acid content in soybean seeds

From 2018 to 2019, the oleic acid content of each soybean line was analysed by SPSS 22.0 software. The oleic acid content of the seeds approached the normal distribution (Fig. 1a, b). The standard deviation (SD) of the oleic acid content of soybean seeds was 6.7 in 2018 and 7.5 in 2019 (Fig. 1c). The normal distribution is a probability function that describes how the values of a variable are distributed. It is a symmetric distribution where most of the observations cluster around the central peak, and the probabilities for values farther away from the mean taper off equally in both directions. The results also indicated that the oleic acid content in different soybean lines is significantly different, and the distribution of the oleic acid content in soybean seeds is continuous, which is consistent with the genetic law of quantitative traits. The oleic acid content in the soybean line q070 was $35.32 \%$ in 2019 and $34.84 \%$ in 2018 , which was the highest among all the soybean lines. The oleic acid content in the soybean line q024 was 5.49\% in 2018 and $6.33 \%$ in 2019, which was the lowest among all the soybean lines.

\section{Soybean fatty acid correlation analysis and heritability calculation}

The multiple linear regression model was used to analyse the relationship between different fatty acids. According to the regression coefficients of the standardized multiple linear regression model, the soybean oleic acid content is significantly negatively correlated with the linoleic acid content (Fig. 2). The correlation coefficient between oleic acid and linoleic acid is -0.660 (Table 1 ). There was a significant positive correlation between soybean oleic acid content and palmitic acid content, with a correlation coefficient of 0.581 . The heritability of the five fatty acids in 260 soybean lines was different, and the heritability of oleic acid was 0.652 (Additional file 1: Table S1).

\section{SNP genotyping and SNP annotation}

In our experiments, SLAF-seq technology was used to sequence soybean genomic DNA. A total of 1,102,987 SLAF tags and 2,311,337 SNP markers were obtained (Fig. 3a, b). The results of SNP distribution on chromosomes are shown in Fig. 3b. According to the location information for SNP loci in the reference genome (CDS regions, gene regions or intergenic regions), the mutated loci (non-synonymous mutations) were predicted. More than $50 \%$ of the SNPs were located in the intergenic regions (stretches of DNA sequences located between genes). Ten percent of the SNP markers were located in the upstream region of genes, and $10 \%$ of the SNPs were 

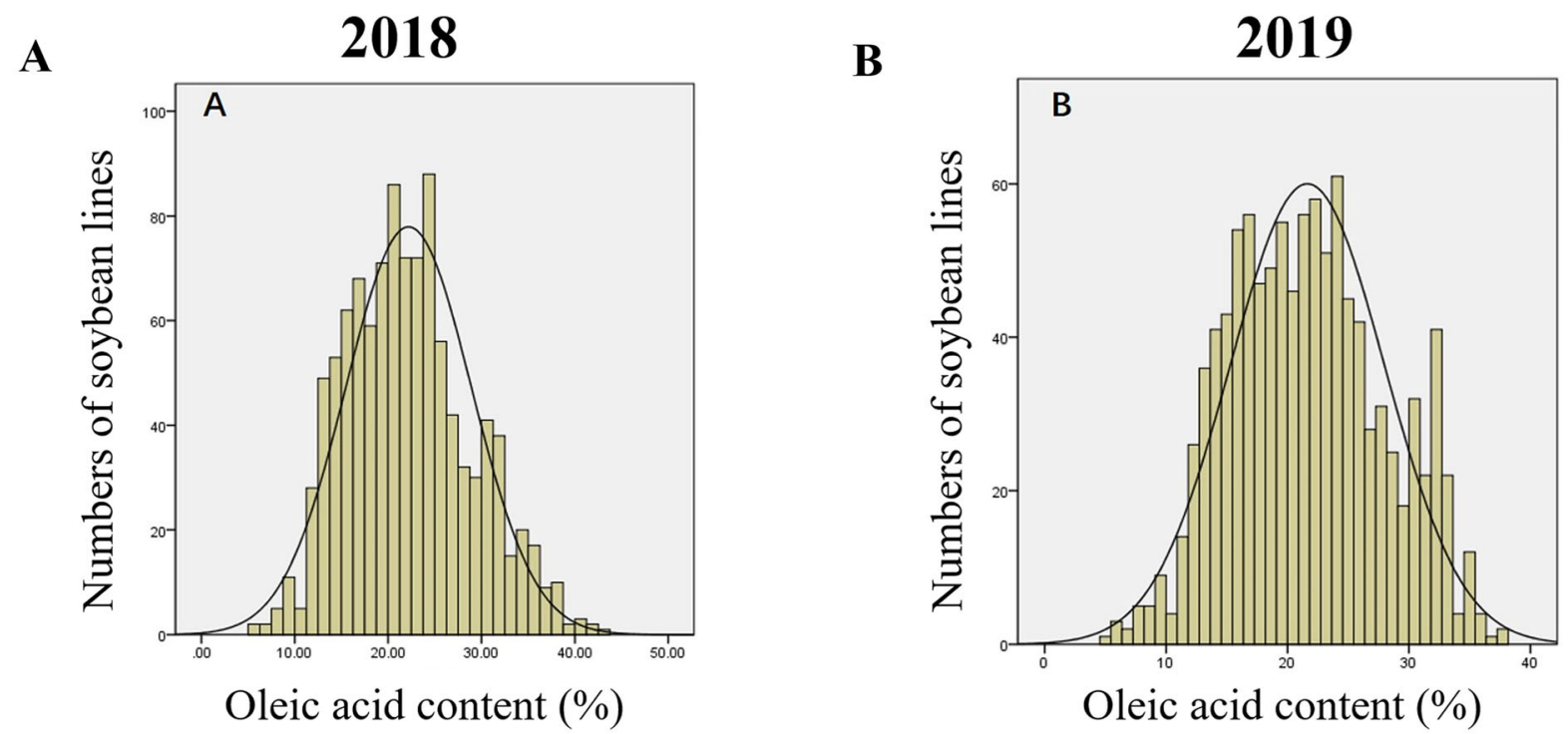

\begin{tabular}{|c|c|c|c|c|c|c|}
\hline Years & Traits & Numbers & minimum & Maximum & Average & Standard devi \\
\hline 2019 & Oleic acid & 780 & 6.33 & 35.32 & 21.3341 & 7. 53547 \\
\hline 2018 & Oleic acid & 780 & 5.49 & 34.84 & 22.1941 & 6.72357 \\
\hline
\end{tabular}

Fig. 1 Frequency distribution of oleic acid content in soybean seeds in 2018 and 2019. The X-axis on the graph shows the range of content of oleic acid. The Y-axis is the number of soybean lines. The distributions of oleic acid content in 2018 (a) and in 2019 (b) resemble the bell-shaped curve for a normal distribution. (c) Statistical analysis of oleic acid content in soybean seeds from 2018 to 2019

located in the downstream region of genes. A total of $4.99 \%$ of the SNP loci were located in the protein-coding regions. Nine percent of the SNP markers were located in introns (Fig. 3c).

\section{Phylogenetic analysis, genetic structure analysis, principal components analysis (PCA)}

From the phylogenetic tree, it can be concluded that the soybean lines originated from two large branches. This result suggests that the 260 soybean lines were from the same ancestor. However, in the process of evolution, they evolved in two directions (Fig. 4a).

Population structure analysis can quantify the number of ancestors of the studied population and infer the source of each sample. It is a cluster analysis method that is currently widely applied and is helpful for understanding the evolutionary process of materials based on SNPs. This experiment was used to analyse the soybean population structure. For the study population, the number of subpopulations preset in this trial was 15 (Fig. 4b). We analysed the data with EIGENSTRAT for the study of 260 soybean lines. It was concluded that the samples that we collected can be represented as an admixture of two ancestral populations (Fig. 4d).

Based on the difference in SNPs, we performed principal component analysis by using EIGENSOFT software for clustering of the 260 soybean materials. The PCA results showed that the 260 soybean lines clustered together in two subgroups (Additional file 2: Fig. S1). PC1, PC2 and PC3 accounted for $36.43,33.82$ and $33.12 \%$, respectively (Fig. 4c).

\section{Genome-wide association study (GWAS) for seed oleic acid content}

Based on the oleic acid content of 260 soybean lines, TASSEL software (Glm model, mlm model, $\mathrm{cmlm}$ model), fastlmmc software, and Emmax software were used for the GWAS. SNP markers significantly correlated with the oleic acid content of soybean seeds were detected, and the linkage disequilibrium (LD) distance was set to $8.9 \mathrm{~kb}$. The Manhattan and QQ (quantilequantile) diagrams for the oleic acid content in 2018 and 2019 are shown in Fig. 5 and Fig. 6. A search was performed for SNP markers that were significantly correlated with the oleic acid content of soybean seeds (Additional file 3: Table S2). In this study, we evaluated 


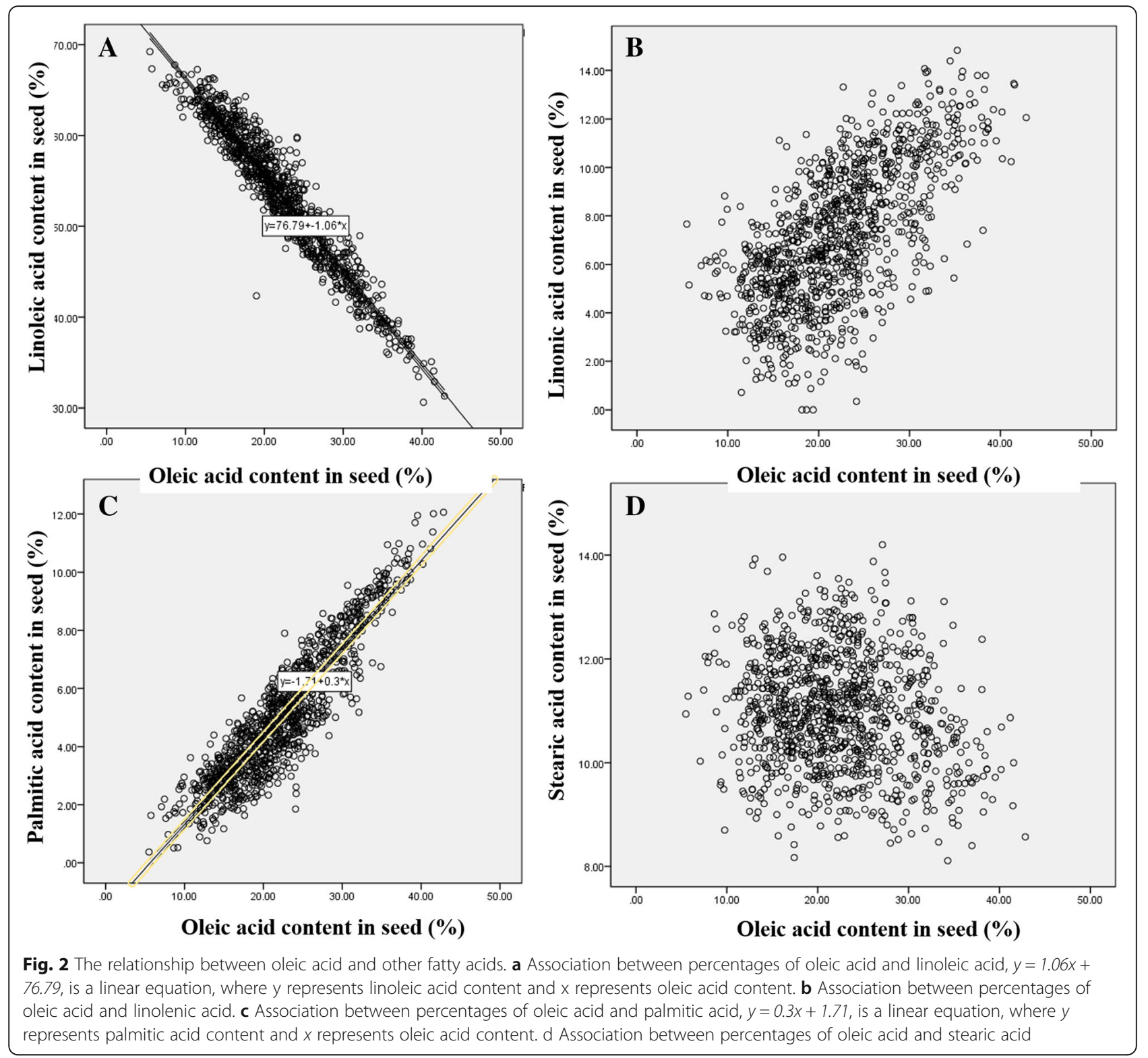

genome-wide LD in 260 accessions and found that the $\mathrm{LD}\left(\mathrm{R}^{2}\right)$ values decayed to half of the maximum value within $9.5 \mathrm{~kb}$ (Additional file 4: Fig. S2). Using $9.7 \mathrm{~kb}$ as the linkage disequilibrium attenuation distance, candidate genes related to soybean oleic acid traits were screened within the LD distance. In 2018, 21 candidate genes related to soybean oleic acid content were screened using genome-wide association analysis (Table 2). In 2019, 8 candidate genes were screened by genome-wide association analysis (Table. 2). Based on

Table 1 Correlations of the relative oleic acid content in soybean

\begin{tabular}{|c|c|c|c|c|c|c|}
\hline & & Oleic acid & Linoleic acid & Linolenic acid & Palmitic acid & Stearic acid \\
\hline Oleic acid & Pearson correlation & 1 & $-0.660^{b}$ & $0.332^{\mathrm{a}}$ & $0.581^{b}$ & $-0.254^{b}$ \\
\hline Linoleic acid & Pearson correlation & $-0.660^{b}$ & 1 & $0.571^{b}$ & $0.051^{b}$ & $-0.893^{b}$ \\
\hline Linolenic acid & Pearson correlation & $0.332^{a}$ & $0.571^{b}$ & 1 & $0.447^{b}$ & $-0.473^{b}$ \\
\hline Palmitic acid & Pearson correlation & $0.581^{b}$ & $0.051^{b}$ & $0.447^{b}$ & 1 & $-0.043^{b}$ \\
\hline Stearic acid & Pearson correlation & $-0.254^{a}$ & $-0.893^{b}$ & $-0.473^{b}$ & $-0.043^{b}$ & 1 \\
\hline
\end{tabular}

Note: ${ }^{\mathrm{a}}$ indicates significance at $0.05,{ }^{\mathrm{b}}$ indicates significance at 0.01 


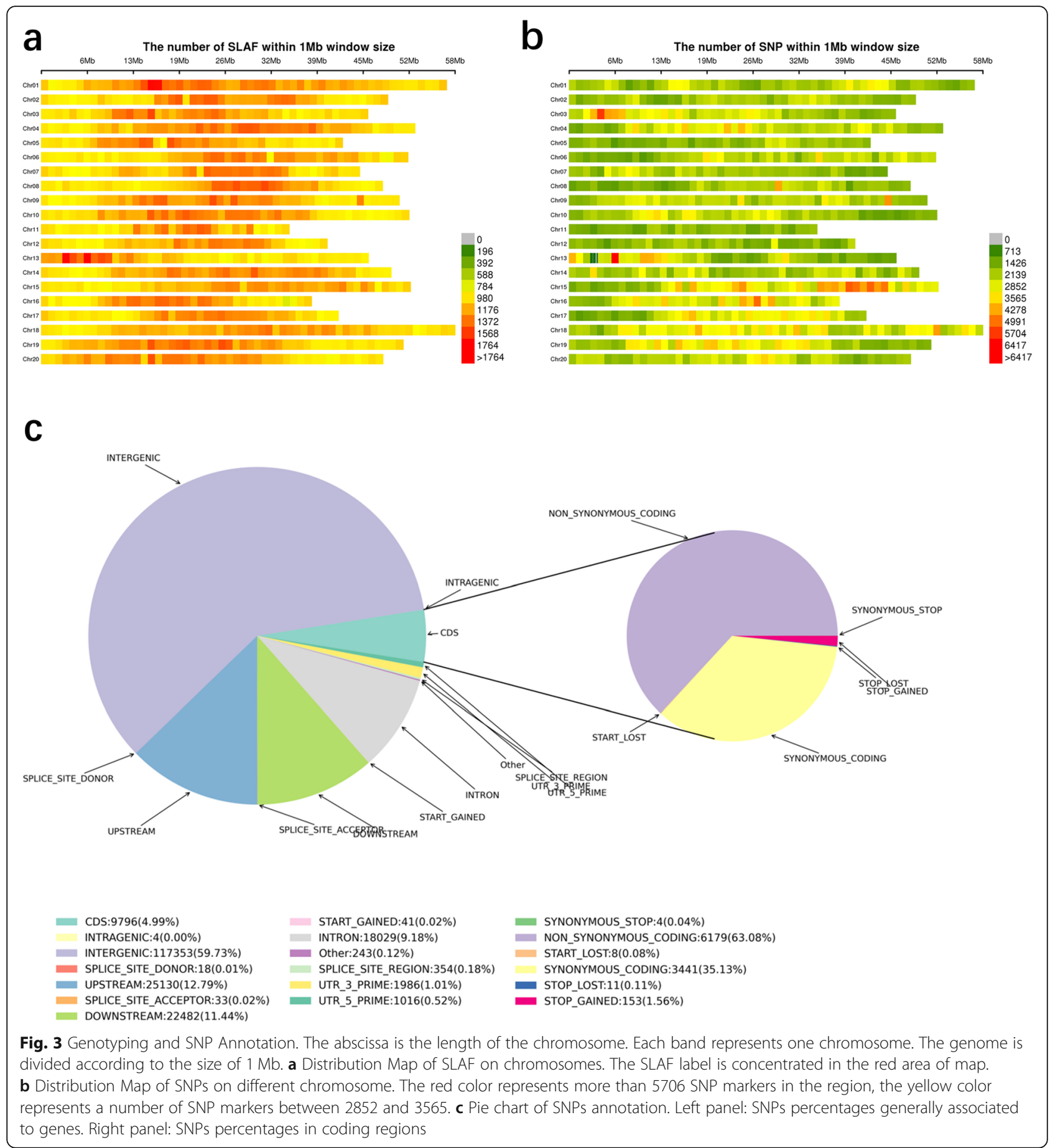

GO terms, the functions of the genes were as follows: 1 . major $\mathrm{CHO}$ metabolism (GO:0004527), 2. cell wall (GO: 0009058), 3. lipid metabolism (GO:0005737), 4. metal ion binding (GO:0046872), 5. N metabolism (GO:0006499), 6. amino acid metabolism (GO:0008152), 7. secondary metabolism (GO:0016491), 8. stress (GO:0034976), 9. redox (ECO:0000313), 10. misc. (GO:0004526), 11. protein (GO: 0004674), 12. cell (GO:0051510), 13. signalling (GO:
0009862), 14. development (GO:0007275), and 15. transport (GO:0003712). The functional distribution of candidate genes is shown in Fig. 7. The first promising candidate gene, Glyma.11G229600.1, located on chromosome 11, was detected by GWAS during 2018 and 2019. Its function was not annotated in the soybean database. According to Swissprot annotation, the Glyma.11G229600.1 gene belongs to the plant BAG protein family. The second promising 


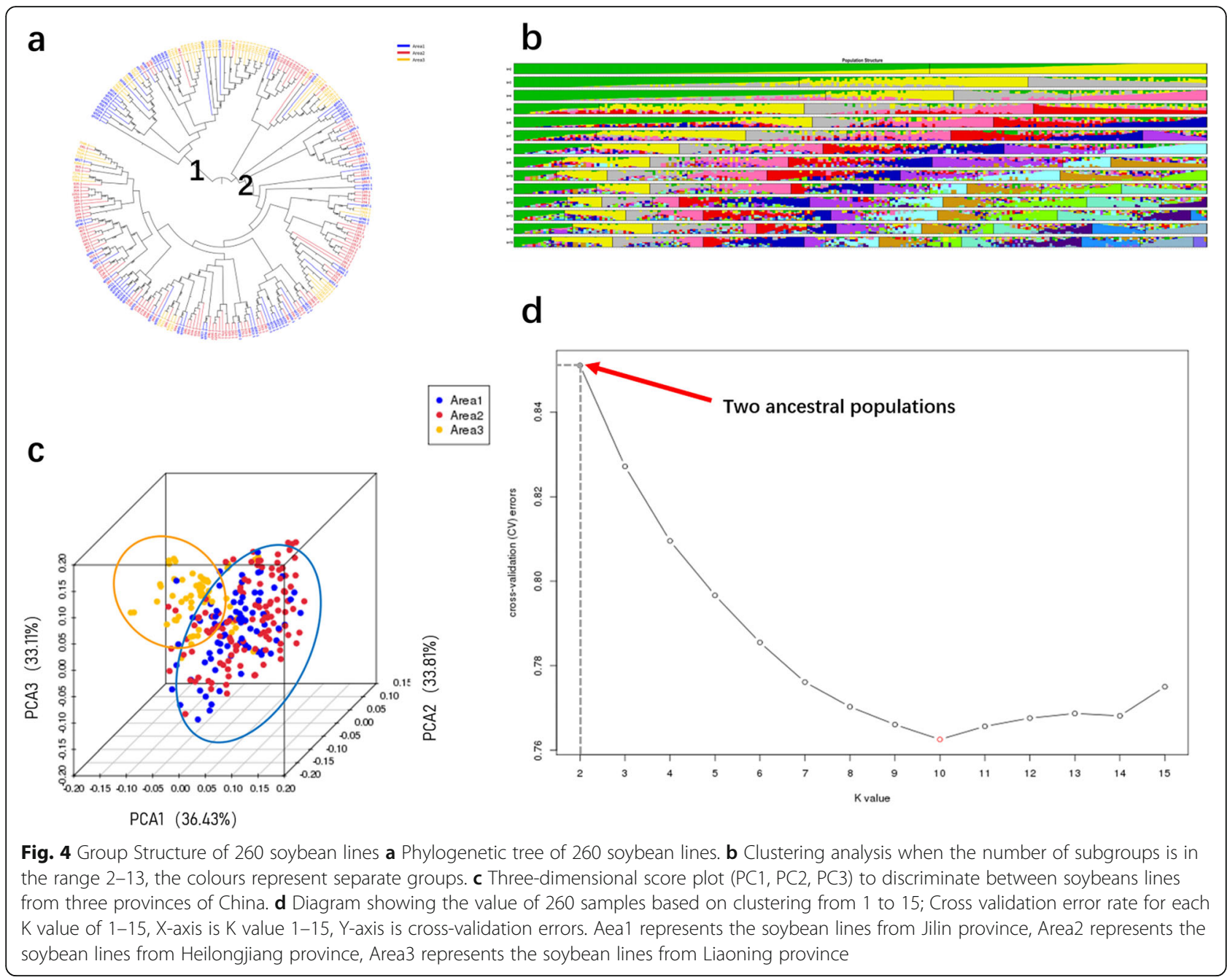

candidate gene, Glyma.04G102900.1, located on chromosome 4 , was also detected by GWAS in the 2 years. Again, there is no report regarding the function of this candidate gene in the soybean database, according to Swissprot annotation. A similar gene in Arabidopsis belongs to the plant GRAS protein family.

\section{Expression of two candidate genes in different tissues}

To validate their association with oleic acid content, the expression of the two selected candidate genes was measured in different tissues (root, stem, leaf and seed) by using qRT-PCR. The lectin gene (GenBank: A5547-127) was used as the reference gene. The results showed that the candidate gene Glyma.11G229600.1 in soybean seedlings was expressed in different tissues, but the relative expression level of the gene was differed significantly, ranging from 1.23 to 4.31 in soybean leaves, from 10.21 to 39.56 in stems, and from 16.21 to 43.14 in roots (Fig. 8a). The candidate gene Glyma.11G229600.1 had the lowest relative expression level (1.23) in the leaves of the soybean line q001, which has the lowest oleic acid content. The relative expression level of Glyma.11G229600.1 in the seeds of the soybean line q001 was also the lowest (25.26). The relative expression level of the candidate gene Glyma.11G229600.1 in the leaves of the soybean line q353 was 4.3 times higher than that in the leaves of the soybean line q001, and the level in seeds was 10 times higher than that in leaves (Additional file 5: Fig. S3). In general, the correlation coefficient between Glyma.11G229600.1 and the oleic acid content was 0.980-0.994 $(P<0.01)$ (Additional file 6: Table. S3). This result strongly indicates that the candidate gene Glyma.11G229600.1 plays a positive role in regulating the oleic acid content in seeds.

The relative expression of Glyma.04G102900.1 was also analysed in soybean leaves, stems, roots and seeds. The relative expression level of Glyma.04G102900.1 in leaves ranged from 9.62 to 44.41 and that in stems ranged from 3.18 to 28.11 (Fig. 8b). Specifically, in the soybean line q001, which has the lowest oleic acid content in seeds, the candidate gene Glyma.04G102900.1 showed the highest expression level, which was 49.01 (Additional file 7: Fig. S4). In general, the relative expression level of Glyma.04G102900.1 in different tissues differed significantly 


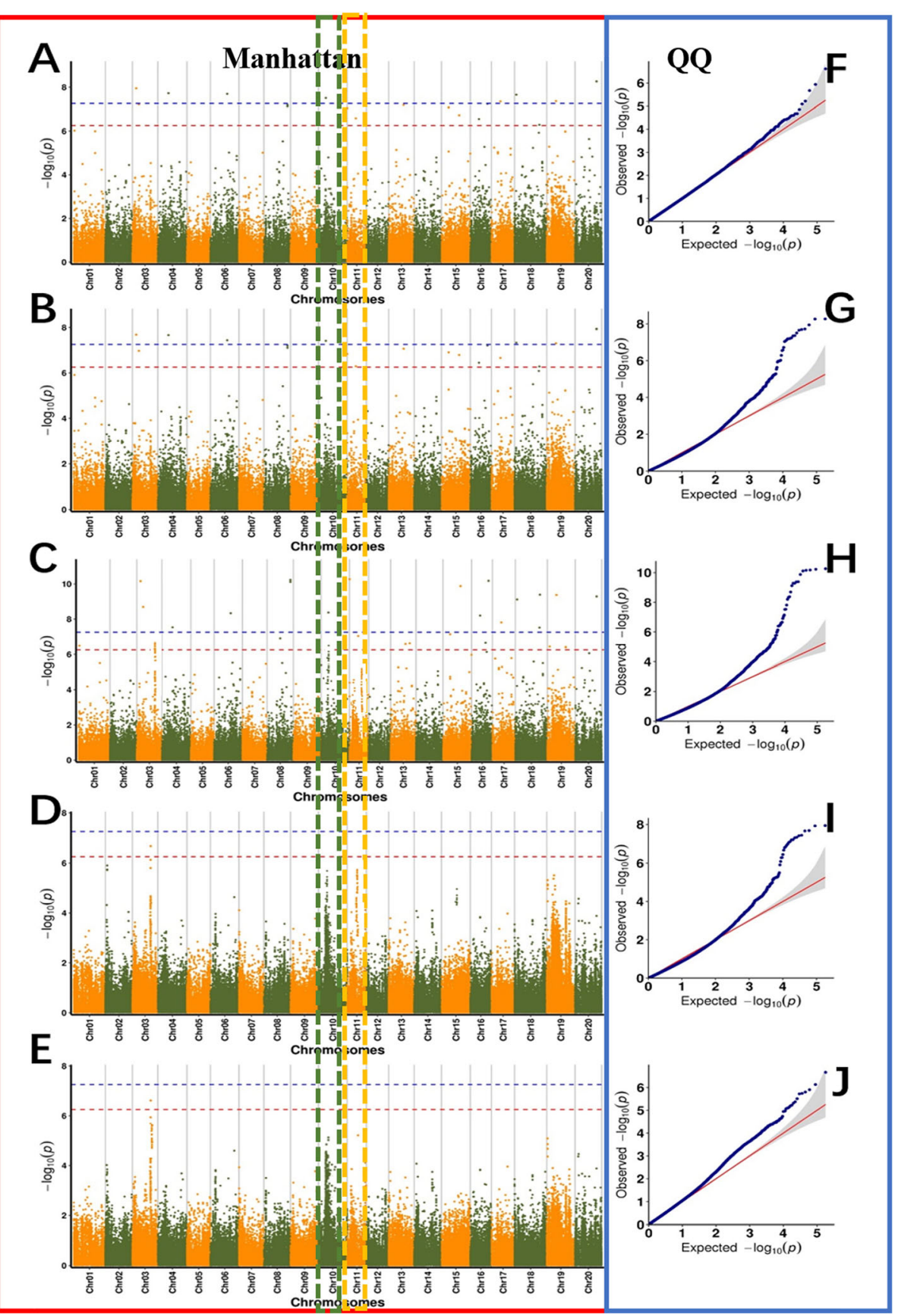

Fig. 5 Genome-wide Manhattan plots of associations to oleic acid content in the 2018 analysis. In the left panel, the X-axis indicates the SNPs along each chromosome; the Y-axis is the - $\log 10$ (P-value) for the association, the threshold value was set at $-\log (p)>6.20$ (red) and $-\log (p)>7.20$ (blue). a to e are GWAS results based on the cmlm model, EmMax model, fastlmm model, GLM, and MLM are in F to J, respectively. e GWAS result based on the MLM model. Q-Q plots for oleic acid using cmlm model (f), EmMax model (g), fastlmm model (h), GLM (I), MLM (j). The grey area represents the 95\% concentration band. Each dot represents a SNP

$(P<0.05)$, with a correlation coefficient between Glyma.04G102900.1 and oleic acid content of $-0.964 \sim$ - 0.998 (Additional file 8: Table. S4). This result indicates that Glyma.04G102900.1 is closely related to the oleic acid content, specifically showing a negative effect on the oleic acid content in seeds.

\section{Discussion}

Soybean oil is composed of five fatty acids: palmitic acid $(16: 0)$, stearic acid (18:0), oleic acid (18:1), linoleic acid (18:2), and linolenic acid (18:3) [17], and the percentage of these five fatty acids in soybean oil averages 10, 4, 18, 55 , and $13 \%$, respectively. It was found that the oleic acid 


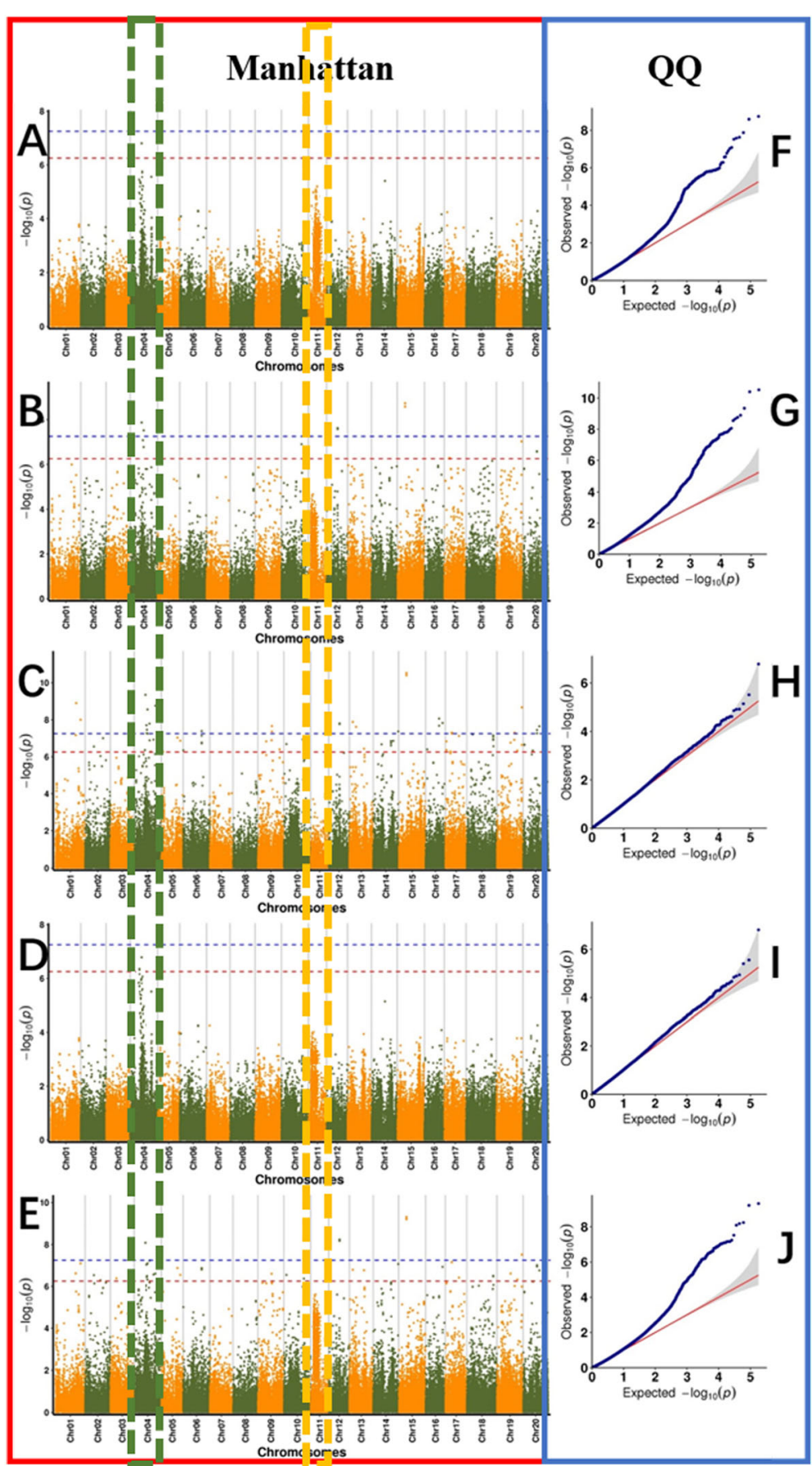

Fig. 6 Genome-wide Manhattan plots of associations for oleic acid content for 2019 analysis. In the left panel, the X-axis indicates the SNPs along each chromosome; the $Y$-axis is the $-\log 10$ (P-value) for the association, the threshold value was set at $-\log (p)>6.20($ red) and $-\log (p)>7.20$ (blue). a GWAS result based on the cmlm model, $\mathbf{b}$ GWAS result based on the EmMax model, $\mathbf{c}$ GWAS result based on the fastlmm model, $\mathbf{d}$ GWAS result based on the GLM model, e GWAS result based on the MLM model. Q-Q plots for oleic acid using cmlm model (f), EmMax model (g), fastlmm model (h), GLM (I), MLM (j). The grey area represents the 95\% concentration band. Each dot represents a SNP

content in grains of different soybean varieties varied greatly [18]. Japanese scientists collected 319 Japanese soybean varieties in 2016; among the 319 accessions, the oleic acid content in seeds ranged from 7.66 to $15.86 \%$, and 101 accessions had seed oleic acid levels of $11.5 \%$
[19]. In 2008, cultivated and wild soybean germplasms from different regions were analysed for their fatty acid content. The results showed that the average fat content in cultivated soybean was $17.21 \%$, which was $6.22 \%$ higher than that in wild soybean; the oleic acid content 
Table 2 Correlation between relative gene expression and oleic acid content in soybean

\begin{tabular}{|c|c|c|c|c|c|}
\hline Year & Chromosome & Gene & Predicted function & Length & $\begin{array}{l}\text { Contribution } \\
\text { rate }\end{array}$ \\
\hline \multirow[t]{21}{*}{2018} & \multirow[t]{2}{*}{ Chr03 } & Glyma.03G054100.1 & $\begin{array}{l}\text { 3PREDICTED: Glycine max TMV resistance protein N-like (LOC100805036), transcript } \\
\text { variant X3, mRNA }\end{array}$ & 687 & 0.10 \\
\hline & & Glyma.03G168200.3 & $\begin{array}{l}\text { 3PREDICTED: Glycine max pleiotropic drug resistance protein 1-like } \\
\text { (LOC100791601), mRNA }\end{array}$ & 4662 & 0.07 \\
\hline & \multirow[t]{3}{*}{ Chr04 } & Glyma.04G191100.1 & \multirow[t]{2}{*}{ 3PREDICTED: Glycine max probable pectate lyase 18-like (LOC100814679), mRNA } & 1657 & 0.32 \\
\hline & & Glyma.04G102900.1 & & 2522 & 0.43 \\
\hline & & Glyma.04G203200.1 & $\begin{array}{l}\text { 3PREDICTED: Glycine max respiratory burst oxidase homolog protein C-like } \\
\text { (LOC100800248), mRNA }\end{array}$ & 2440 & 0.08 \\
\hline & Chr05 & Glyma.05G155300.1 & $\begin{array}{l}\text { 3PREDICTED: Glycine max ATP carrier protein 2, chloroplastic-like (LOC100797684), } \\
\text { mRNA }\end{array}$ & 1655 & 0.11 \\
\hline & \multirow[t]{2}{*}{ Chr07 } & Glyma.07G033100.1 & $\begin{array}{l}\text { 3PREDICTED: Glycine max ADP,ATP carrier protein 1, chloroplastic-like } \\
\text { (LOC100793284), mRNA }\end{array}$ & 2317 & 0.12 \\
\hline & & Glyma.07G089000.1 & $\begin{array}{l}\text { 3PREDICTED: Glycine max VIN3-like protein 1-like (LOC100780157), transcript variant } \\
\text { X2, mRNA }\end{array}$ & 2756 & 0.10 \\
\hline & \multirow[t]{2}{*}{ Chr08 } & Glyma.08G019700.1 & $\begin{array}{l}\text { 3PREDICTED: Glycine max calcium-dependent protein kinase 3-like } \\
\text { (LOC100777096), transcript variant 1, mRNA }\end{array}$ & 1877 & 0.16 \\
\hline & & Glyma.08G185000.2 & $\begin{array}{l}\text { 3PREDICTED: Glycine max probable plastid-lipid-associated protein 4, chloroplastic- } \\
\text { like (LOC100803367), transcript variant 1, mRNA }\end{array}$ & 979 & 0.15 \\
\hline & Chr11 & Glyma.11G229600.1 & $\begin{array}{l}\text { 3PREDICTED: Glycine max DNA replication complex BAG protein, transcript variant } \\
\text { 2, mRNA, }\end{array}$ & 1257 & 0.47 \\
\hline & Chr13 & Glyma.13G163400.1 & $\begin{array}{l}\text { 3PREDICTED: Glycine max protein S-acyltransferase 24-like (LOC100777470), misc_ } \\
\text { RNA }\end{array}$ & 2490 & 0.32 \\
\hline & Chr14 & Glyma.14G045100.1 & 3PREDICTED: Glycine max abscisic-aldehyde oxidase-like (LOC100812604), mRNA & 4517 & 0.22 \\
\hline & \multirow[t]{8}{*}{ Chr15 } & Glyma.15G117700.1 & 3PREDICTED: Glycine max uncharacterized LOC102666654 (LOC102666654), mRNA & 693 & 0.17 \\
\hline & & Glyma.15G120100.1 & $\begin{array}{l}\text { 3PREDICTED: Glycine max tRNA methyltransferase } 10 \text { homolog A-like } \\
\text { (LOC100779099), mRNA }\end{array}$ & 1337 & 0.10 \\
\hline & & Glyma.15G120200.2 & 3PREDICTED: Glycine max uncharacterized LOC102665381 (LOC102665381), mRNA & 1227 & 0.08 \\
\hline & & Glyma.15G127500.1 & 3PREDICTED: Glycine max polygalacturonase-like (LOC100785701), mRNA & 1551 & 0.10 \\
\hline & & Glyma.15G201700.1 & 3PREDICTED: Glycine max uncharacterized LOC100814752 (LOC100814752), mRNA & 1945 & 0.11 \\
\hline & & Glyma.15G210100.3 & $\begin{array}{l}\text { 3PREDICTED: Glycine max alpha,alpha-trehalose-phosphate synthase [UDP-forming] } \\
\text { 1-like (LOC100797320), transcript variant X6, mRNA }\end{array}$ & 3542 & 0.12 \\
\hline & & Glyma.15G244000.1 & 3PREDICTED: Glycine max uncharacterized LOC100814749 (LOC100814749), mRNA & 1213 & 0.10 \\
\hline & & Glyma.15G261100.1 & $\begin{array}{l}\text { 3PREDICTED: Glycine max uncharacterized LOC100801946 (LOC100801946), } \\
\text { transcript variant X1, mRNA }\end{array}$ & 3888 & 0.09 \\
\hline \multirow[t]{8}{*}{2019} & Chr19 & Glyma.19G110600.1 & 3PREDICTED: Glycine max uncharacterized LOC102659858 (LOC102659858), mRNA & 1709 & 0.10 \\
\hline & Chr02 & Glyma.02G220300.1 & 2PREDICTED: Glycine max ataxin-2-like (LOC100788042), mRNA & 1135 & 0.18 \\
\hline & Chr04 & Glyma.04G102900.1 & & 2522 & 0.10 \\
\hline & Chr08 & Glyma.08G071600.1 & $\begin{array}{l}\text { 2PREDICTED: Glycine max metacaspase-3-like (LOC100796113), transcript variant X2, } \\
\text { mRNA }\end{array}$ & 1839 & 0.12 \\
\hline & Chr11 & Glyma.11G229600.1 & $\begin{array}{l}\text { 3PREDICTED: Glycine max DNA replication complex BAG protein, transcript variant } \\
\text { 2, mRNA, }\end{array}$ & 1257 & 0.47 \\
\hline & \multirow[t]{2}{*}{ Chr12 } & Glyma.12G224000.1 & 2PREDICTED: Glycine max uncharacterized LOC102660202 (LOC102660202), mRNA & 2799 & 0.17 \\
\hline & & Glyma.12G227300.1 & 2PREDICTED: Glycine max DNA ligase 1-like (LOC100818049), mRNA & 2728 & 0.17 \\
\hline & Chr20 & Glyma.20G026100.1 & $\begin{array}{l}\text { 2PREDICTED: Glycine max 26S proteasome non-ATPase regulatory subunit } 7 \text { homo- } \\
\text { log A-like (LOC100816479), mRNA }\end{array}$ & 896 & 0.08 \\
\hline
\end{tabular}

in cultivated soybean was $23.25 \%$, which was $7.75 \%$ higher than that in wild soybean; and the linoleic acid content was $53.53 \%$, which was $2.57 \%$ lower than that in wild soybean [20]. In this study, the oleic acid content ranged from 13.5 to $38.4 \%$. The results also showed that the average oleic acid content in soybean germplasms varied greatly, and the oleic acid content in soybean grain was significantly different between regions. In 

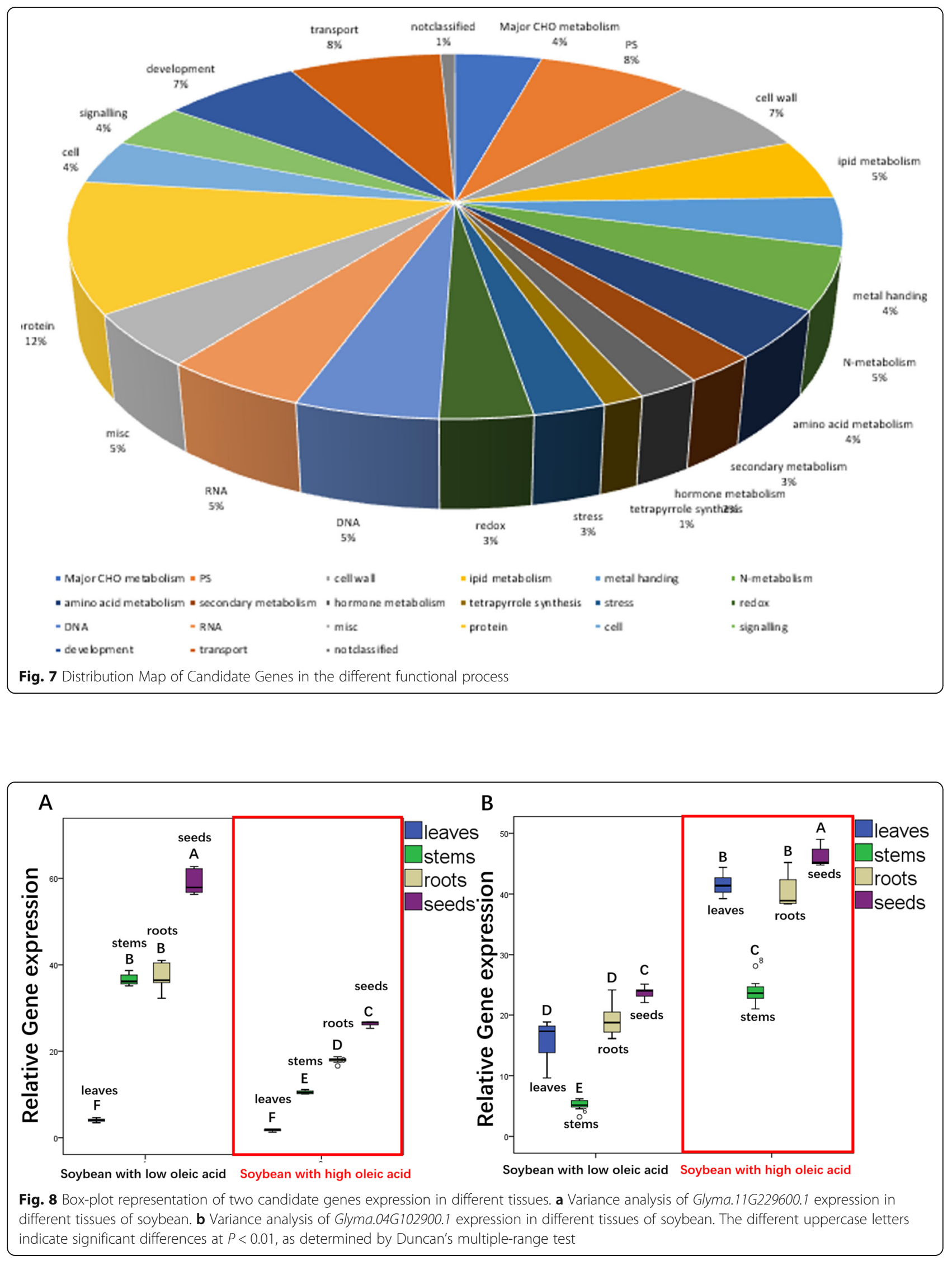
Kurt's study, the correlation analysis clearly indicated a significant and negative correlation of oleic acid with linoleic acid $(r=-0.701, P<0.0001)$ and stearic acid $(r=$ $-0.218, P<0.001)$, stearic acid was significantly positively correlated with oleic and arachidic acid, while stearic acid had an inverse association with both linoleic and linolenic acids [21]. In our study, the oleic acid content was also significantly positively correlated with linoleic acid (0.454). The results indicated that the relationship between oleic and linoleic acids may be helpful in evaluating varieties that are rich in oleic acid.

Glyma.11G229600.1, located on chromosome 11, was simultaneously detected by GWAS during 2018 and 2017. Glyma.11G229600.1 belongs to the plant BAG protein family. The BAG proteins are a broadly conserved gene family with homologs spanning wide evolutionary distances, including yeast, animals, and plants [22]. Studies have shown that BAG proteins are also present in Arabidopsis thaliana [23]. The BAG protein family plays an important role in plant growth and development. Overexpression of BAG7 can increase plant sensitivity to temperature, and BAG4 encodes antiapoptotic genes that have been reported to confer tolerance to salinity and drought stresses in transgenic tobacco [24]. Drought treatment at different growth stages also contributed to differences in fatty acids [25]. The fatty acid composition and amino acid composition were significantly affected by drought stress [26]. Severe drought increased the protein content by 4.4 percentage points, while the oil content decreased by 2.9 percentage points. With increasing drought stress, measured as accumulating stress over days, the protein content increased linearly, and the oil content decreased [27]. It can be speculated that Glyma.11G229600.1 may increase the drought tolerance of soybean, thus affecting the accumulation of oleic acid in soybean seeds.

Glyma.04G102900.1 belongs to the plant GRAS protein family. GRAS proteins constitute an important family of plant-specific proteins named after the first three members discovered: gibberellic acid insensitive (GAI), repressor of gai (RGA) and scarecrow (SCR). At least 33 GRAS genes have been identified in A. thaliana and rice [28]. Two GRAS domain proteins have recently been discovered in pulses [29]. Rhizobial bacteria enter a symbiotic interaction with pulses, activating diverse responses in roots through the lipochito oligosaccharide signalling molecule Nod factor. Indeed, a study showed that a GRAS protein transduces calcium signals in plants and acts as a possible regulator of Nod-factor-inducible gene expression [30]. In this study, we investigated the expression of two candidate genes in different independent soybean lines by qRT-PCR and found that the expression of the candidate genes varied in these lines. We discovered two genes that were correlated with oleic acid content in soybean seed in both the 2018 and 2019 analyses. This is the first time that the key genes Glyma.04G102900.1 and Glyma.11G229600.1 have been reported to be associated with the oleic acid content. Hence, further studies should be conducted to support this finding. Our results provide a basis for deciphering the mechanism underlying the determination of fatty acid composition in soybean. Moreover, the SNP markers identified here demonstrate that marker-assisted selection is a powerful strategy for identifying genes of interest in soybean and can be used in breeding programmes aimed at optimizing fatty acid profiles in seeds.

\section{Conclusions}

In this study, the genome-wide association study (GWAS) technique was used to find SNP markers correlated with oleic acid content. In 2018, 20 new candidate genes related to oleic acid content were detected, and in 2018, a total of 8 new candidate genes related to oleic acid content were also detected. Glyma.04G102900.1 on chromosome 4 and Glyma.11G229600.1 on chromosome 11 were detected in both analyses (2018 and 2019). Glyma.04G102900.1 and Glyma.11G229600.1 are new key candidate genes related to oleic acid in soybean seeds.

\section{Methods}

\section{Plant materials}

The 260 soybean materials provided by the Biotechnology Center of Jilin Agricultural University were planted in the experimental field of Jilin Agricultural University (Changchun, China) from 2018 to 2019 (total of 2 years). A randomized complete block design was used. Each soybean line was examined using three biological replicates. The field was divided into three blocks of $260 \mathrm{~m}^{2}$ $(26 \times 10 \mathrm{~m})$, and each block was subdivided into eight sections. Each section was subdivided into 260 subsections. Natural drying (sunlight) was allowed to occur, and then, the seeds were threshed for oleic acid determination. Fourteen soybean varieties with significantly different oleic acid levels were selected to test candidate gene expression. The names of the soybean lines and the fatty acid content are shown in Table 3.

\section{Determination of fatty acid levels in soybean seeds}

The levels of oleic acid and four other fatty acids (stearic acid, palmitic acid, linoleic acid and linolenic acid) in soybean seeds were determined by a NIRSTM DS 2500 instrument (FOSS, Hillerod, Denmark) after harvesting. SPSS version 22.0 software (SPSS Inc., Chicago, IL, USA) was used to calculate the correlation coefficients of fatty acids in soybean seeds. 
Table 3 Names of 14 soybean lines and average levels of five fatty acids

\begin{tabular}{|c|c|c|c|c|c|c|c|c|}
\hline Group & Name of line & Oil & Protein & Oleic acid & Linoleic acid & Linolenic acid & Palmitic acid & Stearic acid \\
\hline \multirow[t]{7}{*}{ Low oleic acid content } & q001 & 21.1 & 37.62 & 9.56 & 67.85 & 10.77 & 9.79 & 1.26 \\
\hline & q024 & 20.18 & 39.1 & 10.42 & 67.19 & 8.57 & 9.02 & 0.75 \\
\hline & q020 & 20.94 & 39.12 & 11.55 & 65.56 & 8.6 & 8.59 & 1.01 \\
\hline & q008 & 17.94 & 43.49 & 11.67 & 64.32 & 7.75 & 9.48 & 1.01 \\
\hline & q035 & 19.13 & 39.17 & 11.99 & 62.45 & 9.43 & 8.73 & 1.38 \\
\hline & T7287 & 20.96 & 37.76 & 12.23 & 60.62 & 7.74 & 8.22 & 2.36 \\
\hline & q033 & 21.1 & 38.03 & 12.4 & 62.92 & 9.33 & 9.86 & 0.81 \\
\hline \multirow[t]{7}{*}{ High oleic acid content } & q318 & 16.88 & 40.29 & 25.34 & 57.71 & 8.17 & 2.32 & 3.27 \\
\hline & q20 & 22.19 & 40.42 & 26.41 & 54.1 & 6.04 & 2.65 & 1.8 \\
\hline & q073 & 21.29 & 41.54 & 28.08 & 50.94 & 7.97 & 1.72 & 2.69 \\
\hline & q176 & 12.36 & 38.91 & 29.73 & 55.13 & 8.85 & 0.76 & 5.06 \\
\hline & q070 & 20.9 & 40.99 & 29.78 & 51.08 & 7.09 & 1.67 & 1.89 \\
\hline & q68 & 16.91 & 39.44 & 29.94 & 53.64 & 6.01 & 0.56 & 4.43 \\
\hline & q353 & 15.59 & 39.49 & 31.02 & 52.18 & 8.6 & 0.99 & 5.73 \\
\hline
\end{tabular}

\section{Genotyping of soybean germplasms}

Total genomic DNA was extracted from the leaves of each soybean line using the CTAB method according to Murray \& Thompson (1980) [31]. The 260 soybean materials were genotyped by specific-locus amplified fragment sequencing (SLAF-seq), and SNP molecular markers were developed. DNA extraction is the first step in sequencing. SNP molecular markers are used for phylogenetic analysis and genetic evolutionary correlation analysis. The restriction endonuclease combination was RsaI-HaeIII. The sequencing service was provided by Beijing Biomarker Biotechnology Company, PR China.

\section{Population structure evaluation}

Principal component analysis (PCA) was used to assess the population structure using the EIGENSOFT software package. Based on the neighbour-joining method, MEGA5 software was used to construct a phylogenetic tree that included each sample.

\section{Genome-wide association study (GWAS)}

Based on the SNP markers obtained by SLAF-Seq technology, the correlation values between SNP markers and oleic acid content were obtained by using the five models (glm, mlm, cmlm, fastlmm and emmax) in TASS EL software. TASSEL software can calculate the Q matrix of the sample population structure according to the $K$ matrix and finally obtain a correlation value for each SNP marker. The results of each model of each trait were annotated based on a 0.000001 level of significance. In this experiment, both the Manhattan map and QQ map were constructed using Haploview software. The Manhattan map was used to represent the correlation between genotype data and phenotypic data. The QQ map was used to represent the level of difference between observed and predicted values. In this study, the candidate genes were predicted by using the Swiss-Prot and NR databases.

\section{Quantitative reverse transcription PCR}

qRT-PCR analysis was performed using a Bio-Rad CFX system (Amersham Biosciences, Little Chalfont, Buckinghamshire, UK). Total RNA was extracted using an Eastep ${ }^{\circ}$ Super Total RNA Extraction Kit (TaKaRa Company, Kusatsu, Shiga, Japan). The amplification reaction conditions were as follows: predenaturation at $95^{\circ} \mathrm{C}$ for $10 \mathrm{~min}$, denaturation at $95^{\circ} \mathrm{C}$ for $10 \mathrm{~s}$, followed by annealing at $53{ }^{\circ} \mathrm{C}$ for $20 \mathrm{~s}$, and extension at $72{ }^{\circ} \mathrm{C}$ for $15 \mathrm{~s}$. The amplification reaction conditions for the Glyma.11G229600.1 gene were as follows: $95^{\circ} \mathrm{C}$ for $10 \mathrm{~min}$; 35 cycles of denaturation at $95^{\circ} \mathrm{C}$ for $30 \mathrm{~s}$, annealing at $67{ }^{\circ} \mathrm{C}$ for $30 \mathrm{~s}$, and extension at $72^{\circ} \mathrm{C}$ for $30 \mathrm{~s}$; and extension at $72^{\circ} \mathrm{C}$ for $10 \mathrm{~min}$. All of the above reactions included 40 cycles. After amplification, the dissolution curve was calculated by the $2^{-\Delta \Delta C t}$ method [32]. The amplification reaction conditions for the gene Glyma.04G102900.1 were as follows: predenaturation at $95^{\circ} \mathrm{C}$ for $10 \mathrm{~min}$; 35 cycles of denaturation at $95^{\circ} \mathrm{C}$ for $30 \mathrm{~s}$, annealing at $59^{\circ} \mathrm{C}$ for $30 \mathrm{~s}$, and extension at $72^{\circ} \mathrm{C}$ for $35 \mathrm{~s}$; and extension at $72^{\circ} \mathrm{C}$ for $10 \mathrm{~min}$. Three biological replicates were used for each gene.

\section{Data analysis}

The phenotypic data were measured and recorded using Microsoft Excel 2010 software. Differential saliency analysis, analysis of variance, correlation analysis and descriptiveness analysis were performed by using SPSS 
19.0 (IBM Corp, Armonk, NY, USA) software [33]. The positive and negative maps and histograms were constructed by using GraphPad Prism software (GraphPad Company, San Diego, CA).

\section{Supplementary information}

Supplementary information accompanies this paper at https://doi.org/10. 1186/s12870-020-02607-W

Additional file $\mathbf{1}$ Table S1. Heritability of fatty acid content in soybean seeds.

Additional file 2 Figure S1. Population structure of the soybean germplasm collection. (PPTX $182 \mathrm{~kb}$ )

Additional file $\mathbf{3}$ Table S2. SNPs identified as being associated with oleic acid content in 2 years $(P<0.000001)$.

Additional file 4 Figure S2. Genome-wide linkage disequilibrium (LD) decay for all 260 accessions. (PPTX $223 \mathrm{~kb}$ )

Additional file 5 Figure S3. Expression of Glyma.11G229600.1 in different tissues of soybean lines. (PPTX $354 \mathrm{~kb}$ )

Additional file 6 Table S3. Correlation between Glyma.11G229600.1 expression and oleic acid content.

Additional file 7 Figure S4. Expression of Glyma.04G102900.11 in different tissues of soybean lines. (PPTX $233 \mathrm{~kb}$ )

Additional file 8 Table S4 Correlation between Glyma.04G102900.1 expression and oleic acid content.

\section{Abbreviations}

SNP: Single-nucleotide polymorphism; SLAF-seq: Specific-locus amplified fragment sequencing; GWAS: Genome-wide association study; LD: Linkage disequilibrium; qRT-PCR: Real-time quantitative PCR; cM: Centimorgan; QTL: Quantitative trait locus; PCA: Principal component analysis; QQ: Quantile-quantile

\section{Acknowledgements}

We are grateful for the generous grant from the Biotechnology Center of Jilin Agricultural University and the Jilin Academy of Agricultural Sciences that made this work possible.

\section{Authors' contributions}

WP and QD designed the experiments. QD and XYL planned and performed the experiments. CM, AP and QD edited the manuscript. All authors discussed the results and commented on the manuscript. All authors have read and approved the manuscript.

\section{Funding}

The project was funded by the National Key R\&D Program for Crop Breeding (2016YFD0100201). The funder took part in the design of the study; collection, analysis and interpretation of the data; and writing of the manuscript.

\section{Availability of data and materials}

The raw sequence information that support the findings of this study are available from [Beijing Biomarker Biotechnology Co., Itd, Beijing, China] but restrictions apply to the availability of these data, which were used under license for the current study, and so are not publicly available. Data are however available from the corresponding authors upon reasonable request and with permission of [Beijing Biomarker Biotechnology Co., Itd, Beijing, China]. Other datasets supporting the conclusions of this article are included within the article and its additional files.

Ethics approval and consent to participate Not applicable.

\section{Consent for publication}

Not applicable.

\section{Competing interests}

The authors declare that they have no competing interests.

\section{Author details}

${ }^{1}$ Research Center of Integrative Medicine, School of Basic Medical Sciences, Guangzhou University of Chinese Medicine, Guangzhou 510006, Guangdong, PR China. ${ }^{2}$ School of Biosciences and Veterinary Medicine, University of Camerino, Camerino, MC, Italy. ${ }^{3}$ Biotechnology Center of Jilin Agricultural University, Jilin Agricultural University, Changchun 130118, PR China.

Received: 11 July 2020 Accepted: 16 August 2020

Published online: 28 August 2020

\section{References}

1. Liu S, Zhang P, Marley B, Liu W. The factors affecting farmers' soybean planting behavior in Heilongjiang Province, China. Agriculture. 2019;9(9):188.

2. Tunde-Akintunde T, Olajide J, Akintunde B. Mass-volume-area related and mechanical properties of soybean as a function of moisture and variety. Int J Food Prop. 2005;8(3):449-56.

3. Zhao X, Chang H, Feng L, Jing Y, Teng W, Qiu L, et al. Genome-wide association mapping and candidate gene analysis for saturated fatty acid content in soybean seed. Plant Breed. 2019:138(5):588-98.

4. Collins F, Sedgwick V. Fatty acid composition of several varieties of soybeans. J Am Oil Chem Soc. 1959;36(12):641-4.

5. Patil S, Balu D, Melrose J, Chan C. Brain region-specificity of palmitic acidinduced abnormalities associated with Alzheimer's disease. BMC Res Notes. 2008;1(1):20.

6. Mensink RP, Zock PL, Kester AD, Katan MB. Effects of dietary fatty acids and carbohydrates on the ratio of serum total to $\mathrm{HDL}$ cholesterol and on serum lipids and apolipoproteins: a meta-analysis of 60 controlled trials. Am J Clin Nutr. 2003:77(5):1146-55

7. Sherwin E. Oxidation and antioxidants in fat and oil processing. J Am Oil Chem Soc. 1978:55(11):809-14.

8. Combs R, Bilyeu KD. Novel alleles of FAD2-1A induce high levels of oleic acid in soybean oil. Mol Breeding. 2019;39(6):1-11.

9. Wang X, Pang Y, Zhang J, Wu Z, Chen K, Ali J, Ye G, Xu J, Li Z. Genomewide and gene-based association mapping for rice eating and cooking characteristics and protein content. Sci Rep. 2017;7(1):1-10

10. Verslues PE, Lasky JR, Juenger TE, Liu TW, Kumar MN. Genome-wide association mapping combined with reverse genetics identifies new effectors of low water potential-induced proline accumulation in Arabidopsis. Plant Physiol. 2014;164(1):144-59.

11. Brachi B, Morris GP, Borevitz JO. Genome-wide association studies in plants: the missing heritability is in the field. Genome Biol. 2011:12(10):232.

12. Hwang EY, Song Q, Jia G, Specht JE, Hyten DL, Costa J, et al. A genomewide association study of seed protein and oil content in soybean. BMC Genomics. 2014;15(1):1.

13. Cao Y, Li S, Wang Z, Chang F, Kong J, Gai J, et al. Identification of major quantitative trait loci for seed oil content in soybeans by combining linkage and genome-wide association mapping. Front Plant Sci. 2017:8:1222.

14. Bastien M, Sonah H, Belzile F. Genome wide association mapping of Sclerotinia sclerotiorum resistance in soybean with a genotyping-bysequencing approach. Plant Genome. 2014;7:1

15. Kaler AS, Ray JD, Schapaugh WT, King CA, Purcell LC. Genome-wide association mapping of canopy wilting in diverse soybean genotypes. Theor Appl Gent. 2017;130(10):2203-17

16. Zhang J, Wang X, Lu Y, Bhusal SJ, Song Q, Cregan PB, et al. Genome-wide scan for seed composition provides insights into soybean quality improvement and the impacts of domestication and breeding. Mol Plant. 2018;11(3):460-72

17. Priolli RH, Campos JB, Stabellini NS, Pinheiro JB, Vello NA. Association mapping of oil content and fatty acid components in soybean. Euphytica. 2015;203(1):83-96

18. Diers BW, Shoemaker R. Restriction fragment length polymorphism analysis of soybean fatty acid content. J Am Oil Chem Soc. 1992;69(12):1242-4.

19. Wee CD, Hashiguchi M, Anai T, Suzuki A, Akashi R. Fatty acid composition and distribution in wild soybean (Glycine soja) seeds collected in Japan. Asian J Plant Sci. 2017:16:52-64.

20. Pantalone VR, Rebetzke GJ, Wilson RF, Burton JW. Relationship between seed mass and linolenic acid in progeny of crosses between cultivated and wild soybean. J Am Oil Chem Soc. 1997;74(5):563-8. 
21. Kurt C. Variation in oil content and fatty acid composition of sesame accessions from different origins. Grasas Aceites. 2018;69(1):241.

22. Williams B, Kabbage M, Britt R, Dickman MB. AtBAG7, an Arabidopsis BCl-2associated athanogene, resides in the endoplasmic reticulum and is involved in the unfolded protein response. P Natl A Sci. 2010;107(13):6088-93.

23. Yan J, He C, Zhang H. The BAG-family proteins in Arabidopsis thaliana. Plant Sci. 2003;165(1):1-7.

24. Hoang TM, Moghaddam L, Williams B, Khanna H, Dale J, Mundree SG. Development of salinity tolerance in rice by constitutive-overexpression of genes involved in the regulation of programmed cell death. Front Plant Sci. 2015;6:175.

25. Sánchez-Martín J, Canales FJ, Tweed JK, Lee MR, Rubiales D, GómezCadenas A, et al. Fatty acid profile changes during gradual soil water depletion in oats suggests a role for jasmonates in coping with drought. Front Plant Sci. 2018:9:1077.

26. Dwivedi S, Nigam S, Rao RN, Singh U, Rao K. Effect of drought on oil, fatty acids and protein contents of groundnut (Arachis hypogaea L.) seeds. Field crops res. 1996;48(2-3):125-33.

27. Dornbos D, Mullen R. Soybean seed protein and oil contents and fatty acid composition adjustments by drought and temperature. J Am Oil Chem Soc. 1992;69(3):228-31.

28. Hirsch S, Oldroyd GE. GRAS-domain transcription factors that regulate plant development. Plant Signal Behav. 2009;4(8):698-700.

29. Heckmann AB, Lombardo F, Miwa H, Perry JA, Bunnewell S, Parniske M, et al. Lotus japonicus nodulation requires two GRAS domain regulators, one of which is functionally conserved in a non-legume. Plant Physiol. 2006; 142(4):1739-50.

30. Smit P, Raedts J, Portyanko V, Debellé F, Gough C, Bisseling T, et al. NSP1 of the GRAS protein family is essential for rhizobial nod factor-induced transcription. Science. 2005;308(5729):1789-91.

31. Murray M, Thompson WF. Rapid isolation of high molecular weight plant DNA. Nucleic Acids Res. 1980;8(19):4321-6.

32. Livak KJ, Schmittgen TD. Analysis of relative gene expression data using real-time quantitative PCR and the $2^{-\Delta \Delta C T}$ method. Methods. 2001;25(4): 402-8.

33. Verma JP. Data analysis in management with SPSS software. Springer. Berlin: Science \& Business Media; 2013.

\section{Publisher's Note}

Springer Nature remains neutral with regard to jurisdictional claims in published maps and institutional affiliations.

Ready to submit your research? Choose BMC and benefit from:

- fast, convenient online submission

- thorough peer review by experienced researchers in your field

- rapid publication on acceptance

- support for research data, including large and complex data types

- gold Open Access which fosters wider collaboration and increased citations

- maximum visibility for your research: over $100 \mathrm{M}$ website views per year

At BMC, research is always in progress.

Learn more biomedcentral.com/submissions 